\title{
Alimentos sujeitos à fortificação compulsória com ferro: um estudo com gestantes ${ }^{1}$
}

\author{
Foods subject to mandatory fortification with iron: \\ a study with pregnant women
}

Ivana Aragão Lira VASCONCELOS²

Mariana Helcias CORTES²

Denise Costa COITINHO 2,3

RE S U M O

\section{Objetivo}

Avaliar o consumo de alimentos sujeitos à fortificação compulsória com ferro por gestantes atendidas em consultas de pré-natal do Hospital Universitário de Brasília.

\section{Métodos}

Trata-se de série temporal que comparou dados de 228 pares de gestantes a partir de duas avaliações transversais: em 2004, pré-fortificação e em 2005, um ano após intervenção. Dados gestacionais, socioeconômicos, demográficos, índice de massa corporal e consumo alimentar foram coletados. Este último foi aferido por Questionário Semiquantitativo de Freqüência Alimentar incluindo alimentos à base de farinhas de trigo e de milho.

\section{Resultados}

O consumo per capita diário médio de farinhas foi estimado em 121,7g (98,7-115,8), no $1^{\circ}$ momento, e $119,5 \mathrm{~g}(93,6-109,5)$, no $2^{\circ}$ momento $(p>0,05)$, com maior contribuição da farinha de trigo. Os alimentos mais consumidos, em ambos os momentos, foram: pão francês, biscoitos, bolo, macarrão e cuscuz de milho. As gestantes do estudo receberiam uma média de 5,1 mg de ferro adicional, se a fortificação estivesse ocorrendo como o preconizado pela legislação, que corresponde a 19\% da Ingestão Dietética de Referência.

\section{Conclusão}

Os alimentos sujeitos à fortificação são veículos apropriados em relação ao largo consumo, porém são necessários estudos que avaliem a quantidade adicionada e a biodisponibilidade dos compostos de ferro.

Termos de indexação: Alimentos fortificados. Consumo alimentar. Ferro. Gestantes.

\footnotetext{
1 Artigo elaborado a partir da dissertação de I.A.L. VASCONCELOS, intitulada "Avaliação do consumo de alimentos sujeitos à fortificação compulsória com ferro das gestantes atendidas no pré-natal do Hospital Universitário de Brasília, Distrito Federal". Programa de Pós-Graduação em Nutrição Humana, Universidade de Brasília; 2006

${ }^{2}$ Universidade de Brasília, Faculdade de Ciências da Saúde, Departamento de Nutrição, Programa de Pós-Graduação em Nutrição Humana. Campus Universitário Darcy Ribeiro, Asa Norte, 70910-900, Brasília, DF, Brasil. Correspondência para/Correspondence to: I.A.L. VASCONCELOS.E-mails: <ivanaunb@gmail.com>; <ivana@unb.br>.

3 World Health Organization, Department of Nutrition for Health and Development. Geneva, Switzerland.
} 
150 | I.A.L. VASCONCELOS et al.

\section{A B S T R A C T}

\section{Objective}

The objective of this study was to assess the consumption of foods subject to mandatory fortification with iron by pregnant women visiting the Hospital Universitário de Brasilia for prenatal care.

\section{Methods}

A time-series study that compared 228 paired pregnant women through two cross-sectional assessments: in 2004, before flour fortification, and a year later. Pregnancy, socioeconomic and demographic data, body mass index and food consumption patterns were collected. The latter was determined by applying the Semiquantitative Food Frequency Questionnaire and included foods containing wheat and corn flours.

\section{Results}

The daily per capita consumption of flours was estimated to be $121.7 \mathrm{~g}$ (98.7-115.8) in the first interview before fortification and 119.5g (93.6-109.5) in the second interview after fortification ( $>>0.05)$, with a greater consumption of wheat flour. The most consumed foods before and after fortification were French bread, cookies, cakes, pasta and corn couscous. The studied pregnant women would have received an extra $5.1 \mathrm{mg}$ of iron if fortification had been done according to the legislation, which corresponds to $19 \%$ of the Dietary Reference Intake.

\section{Conclusion}

The foods subject to fortification are appropriate vehicles because of their high consumption yet studies that assess the amount of iron added and the bioavailability of the iron compounds used are needed.

Indexing terms: Food fortified. Food consumption. Iron. Pregnant women.

\section{N T R O D U ÇÃ O}

A deficiência de ferro é o principal fator de risco para o desenvolvimento de anemias nutricionais ${ }^{1,2}$. Um dos grupos populacionais mais vulneráveis às anemias nutricionais são as gestantes, em função da baixa ingestão de ferro relativa ao aumento do requerimento do nutriente nesse estado fisiológico, com prevalências estimadas em $52 \%$ em países não-industrializados e $23 \%$ em países industrializados ${ }^{2}$.

No Brasil, os principais estudos de base populacional encontraram prevalências altas de anemia em gestantes: $35,1 \%^{3}$ no estado de São Paulo; $29,2 \%^{4}$ no município de São Paulo (São Paulo); $25,2 \%^{5}$ e 30,9\% ${ }^{6}$, ambos realizados em Recife (Pernambuco). Essas prevalências indicam, em termos de magnitude na saúde pública, uma situação de anemia categorizada como de moderado nível (quando a prevalência é de 20,0 a $39,9 \%)^{2}$.

Como estratégias para controlar essa carência, a Organização Mundial da Saúde (OMS) recomenda programas de educação nutricional, para diversificar o consumo alimentar da população de risco, combinados com ações de suplementação e de fortificação de alimentos com ferro ${ }^{2}$.

Um aspecto essencial da fortificação de alimentos é a eleição do veículo alimentar. Para que um alimento possa ser um veículo potencial de fortificação, deve ser de baixo custo ${ }^{7}$, de alto consumo pela população-alvo ${ }^{7,8}$ e ter um consumo padrão constante com baixo risco de excesso 7 .

A partir disso, em dezembro de 2002, em caráter urgente, a resolução da diretoria colegiada (RDC) n 344, da Agência Nacional de Vigilância Sanitária (ANVISA), tornou obrigatória a fortificação das farinhas de trigo e de milho no País com um mínimo de 4,2mg de ferro e 150 $\mu \mathrm{g}$ de ácido fólico, para cada $100 \mathrm{~g}$ de farinha. Essa resolução estabeleceu o prazo de 18 meses para que as empresas se adequassem ao novo regulamento, ou seja, até junho de $2004^{9}$.

Então, é imprescindível a avaliação da implementação para aprimoramento dessa nova estratégia, já que a fortificação é indicada como a ação de melhor custo-benefício em longo prazo 
na redução da prevalência de deficiência de ferro². Além disso, um dos pontos-chave para o sucesso dessa intervenção está associado à escolha dos veículos alimentares, cujo consumo ainda não foi avaliado em gestantes. O aprofundamento sobre padrões dietéticos desse grupo vulnerável, portanto, assume grande importância para a saúde pública, uma vez que contribui para o embasamento, o direcionamento, a avaliação e o monitoramento de políticas de alimentação e nutrição ${ }^{10}$.

A partir desses pressupostos, o objetivo deste artigo foi avaliar o consumo de alimentos sujeitos à fortificação compulsória com ferro por gestantes atendidas pelo serviço de atenção pré-natal do Hospital Universitário de Brasília (HUB), no Distrito Federal (DF).

\section{MÉ T O D O S}

Esta investigação faz parte de um estudo mais amplo que objetivou avaliar o impacto da fortificação das farinhas de trigo e de milho com ferro das gestantes atendidas pelo pré-natal do HUB. Trata-se de um estudo de cunho descritivo, que contemplou uma série temporal com duas avaliações, em intervalo de 12 meses. Portanto, foram realizados dois inquéritos transversais com gestantes atendidas no ambulatório de pré-natal do HUB. O primeiro momento, de maio a agosto de 2004, imediatamente anterior à entrada em vigor da nova medida regulatória, permitiu a construção de uma linha de base e o segundo momento, de maio a agosto de 2005, um ano após a implementação da RDC $n^{\circ} 344^{\circ}$, possibilitou a avaliação pós-intervenção.

Cada gestante entrevistada em 2004 foi pareada a uma gestante em 2005 segundo o trimestre gestacional, a idade materna ( $<20$ anos; 20 a 35 anos; e >35 anos) e a condição de risco obstétrico (baixo ou alto) ${ }^{11}$. Este procedimento foi utilizado para um melhor controle destas três variáveis relacionadas ao desfecho principal do estudo - presença de anemia ${ }^{12}$. Um total de 228 pares de gestantes foi investigado. Esta amostra é suficiente para estimar uma diferença de prevalências de anemia entre os dois momentos de, no mínimo, 12\%, com intervalo de confiança de $95 \%$ e poder de $80 \%{ }^{12}$.

A coleta de dados foi feita nos dias de atendimento pré-natal. Esse levantamento incluiu todas as gestantes, presentes naqueles momentos, e que aceitaram participar do estudo, independentemente do número de consultas prévias. Foram excluídas, posteriormente, gestantes com diagnóstico médico comprovado de anemia de outra etiologia (Ex.: anemia falciforme); portadoras de doenças crônicas com interferências hematológicas (Ex.: insuficiência renal crônica, imunodeficiências, insuficiência cardíaca, miocardia chagásica, púrpura trombocitopênica idiopática, trombocitopenia, trombose) e gestantes com gravidez de gemelares $^{12}$.

Para a avaliação antropométrica (peso e altura) foram utilizados uma balança digital Marte ${ }^{\circledR}$, com precisão de $100 \mathrm{~g}$ e capacidade máxima de $150 \mathrm{~kg}$, e um estadiômetro de parede Secca ${ }^{\circledR}$, com precisão de $1 \mathrm{~cm}$. As entrevistadas foram pesadas descalças, dispostas no centro da balança, com roupas leves e medidas descalças, em pé, com os braços estendidos dispostos ao lado e junto ao corpo, cabeça em direção ao horizonte, coluna reta e calcanhares juntos e encostados à parede, técnicas propostas por Vasconcelos $^{13}$.

O índice de massa corporal (IMC) foi calculado e o estado nutricional foi classificado conforme Atalah et al. ${ }^{14}$, a partir da idade gestacional. Foram considerados como $1^{\circ}, 2^{\circ}$ e $3^{\circ}$ trimestres da gravidez os períodos até a $13^{\mathrm{a}}$ semana de gestação, entre a $14^{\mathrm{a}}$ e a $27^{\mathrm{a}}$ semanas e acima da $28^{\mathrm{a}}$ semana, respectivamente ${ }^{15}$.

Foi aplicado um questionário contendo perguntas relativas à gestação atual e anterior (idade gestacional, número de gestações, uso de suplemento de ferro), ao status socioeconômico (renda per capita, renda em salário mínimo, anos de estudos completos), aos dados demográfico (idade) e de consumo alimentar. 
A renda e a escolaridade foram coletadas segundo instrumento utilizado pelo Instituto Brasileiro de Geografia e Estatística (IBGE) no Censo Demográfico $2000^{16}$.

O consumo alimentar habitual pregresso, referente ao último ano, foi aferido por um questionário semiquantitativo de freqüência alimentar (QFA) ${ }^{17}$, adaptado após pré-teste. Foi composto por sete diferentes intervalos de freqüência de ingestão de cada alimento - uma vez por dia, duas ou mais vezes por dia, cinco a seis vezes por semana, duas a quatro vezes por semana, uma vez por semana, uma a três vezes por mês e raramente ou nunca - e 55 itens separados em grupos: leite e derivados, carnes e ovos, óleos e gorduras, petiscos e enlatados, cereais e leguminosas, vegetais e frutas, sobremesas e doces, bebidas ${ }^{17}$. Dentre esses itens, incluíram-se preparações que contivessem os veículos de fortificação: farinhas de trigo e de milho. Para aperfeiçoar a percepção das participantes quanto às medidas caseiras consumidas, utilizou-se um registro fotográfico ${ }^{18}$ durante a entrevista.

A informação obtida com o QFA foi convertida em gramas de alimentos consumidos por dia, por meio de tabelas de medidas caseiras ${ }^{19,20}$. As medidas caseiras que não constavam nas tabelas utilizadas foram conseguidas nos rótulos de produtos industrializados e por medição direta, conforme o método sugerido por Moreira ${ }^{20}$. Para obter a estimativa diária do consumo de nutrientes, foi utilizado o software Nutri Survey ${ }^{21}$. O software não é nacional, porém foram considerados os alimentos de tabelas de composição brasileiras para o cálculo ${ }^{22,23}$.

Além de analisar a dieta de acordo com o consumo de energia e nutrientes, foi observada também a freqüência de consumo dos alimentos. Outras variáveis, como o ferro adicionado aos alimentos sujeitos à fortificação e o ferro de alimentos naturalmente fontes do nutriente, foram também relacionadas para explicar o ferro total consumido.

Para energia, adotaram-se as recomendações do National Research Council24; para pro- teína e carboidratos, os parâmetros da Ingestão Dietética de Referência (IDR) ${ }^{25}$ e, para micronutrientes, as referências adotadas pela ANVISA ${ }^{26}$. A recomendação de ferro, nessa resolução, é a mesma da IDR, ou seja, 27mg/dia para gestantes.

As quantidades de farinha de trigo e de milho, presentes em cada tipo de preparação, foram obtidas a partir de fichas de preparação desenvolvidas no Laboratório de Técnica Dietética da Universidade de Brasília. Para isso, foram pesados todos os ingredientes crus, a receita total cozida e porções em diversos tamanhos. Foram considerados os fatores de cocção ou os índices de absorção e o tipo de cozimento, todos influentes na proporção de farinha de cada preparação.

Os dados foram codificados e digitados. Para a análise de dados utilizaram-se os softwares SPSS versão $13.0^{27}$ e SAS versão 9.128.

$\mathrm{Na}$ análise geral, utilizou-se a estatística descritiva como medidas de freqüência, média, desvio-padrão, mediana e intervalo de confiança de $95 \%$. Apesar de as amostras terem sido pareadas, optou-se por análises destinadas a amostras independentes, uma vez que estas permitem obter resultados mais conservadores. No entanto, as análises para amostras pareadas foram feitas (resultados não disponíveis) e não apresentaram diferença em relação aos testes empregados. Estes consideraram nível de significância de $5 \%$ : " $\mathrm{t}$ " de Student para as variáveis contínuas; teste do qui-quadrado ou teste exato do quiquadrado para variáveis categóricas.

Para relacionar as variáveis que explicaram o ferro total consumido em cada momento, aplicou-se o modelo de regressão linear múltipla com procedimento de seleção de variáveis por stepwise, sendo que a primeira variável a entrar no modelo foi aquela que teve a maior relação com o teor de ferro, e assim sucessivamente, até que nenhuma variável apresentasse nível de significância menor ou igual a 5\%.

Os requisitos éticos exigidos pela resolução 196/96, do Conselho Nacional de Saúde ${ }^{29}$, foram seguidos e o estudo foi aprovado pelo Comitê de 
Ética e Pesquisa da Faculdade de Ciências da Saúde da Universidade de Brasília, em 10 de fevereiro de 2004.

\section{RESULTADOS}

Os dados de trimestre gestacional $\left(1^{\circ}\right.$ trimestre: $16,2 \%$; $2^{\circ}$ trimestre: $43,9 \%$; $3^{\circ}$ trimestre: $39,9 \%$, em ambos os momentos), de risco obstétrico (Sim: 32,5\%; Não: 67,5\%, nos dois momentos) e de idade (Tabela 1) são semelhantes nos dois momentos, tendo em vista terem sido as variáveis utilizadas para o pareamento. Nenhuma das demais variáveis estudadas apresentou diferença entre os dois momentos.

Foram observadas porcentagens importantes de baixo peso ( $1^{\circ}$ momento: $25,9 \%$; $2^{\circ}$ momento: $20,4 \%$ ), de sobrepeso ( $1^{\circ}$ momento: $16,7 \%$; $2^{\circ}$ momento: $23,4 \%$ ) e de obesidade ( $1^{\circ}$ momento: $8,3 \%$; $2^{\circ}$ momento: $\left.11,5 \%\right)$. Apesar das diferenças entre as porcentagens, não houve diferença significante do estado nutricional entre os dois momentos do estudo (teste exato do qui-quadrado, $p=0,089$ ). O ferro suplementar foi utilizado por $34,0 \%$ das gestantes, segundo o próprio relato, em ambos os momentos.

Estimou-se a quantidade de ferro adicional proveniente da fortificação, assumindo o valor mínimo indicado pela legislação de 4,2mg por $100 \mathrm{~g}$ de farinha (ferro teórico adicional).

Essas estimativas foram necessárias para o cálculo do consumo per capita total e das farinhas provenientes dos alimentos sujeitos à fortificação e para o cálculo do ferro total (ferro dietético + ferro teórico adicional) no $2^{\circ}$ momento do estudo, quando a fortificação já era compulsória.

O consumo médio total e a contribuição estimada das farinhas são apresentados na Tabela 2. Para a apresentação desses dados, os alimentos que contivessem farinhas de trigo e de milho foram agrupados e classificados segundo o tipo. A categoria pães de trigo engloba as farinhas de trigo provenientes do pão integral, pão francês, pão de fôrma, pão do sanduíche e outros tipos; a categoria biscoitos abrange a farinha de trigo dos biscoitos salgado, doce e recheado; macarrão inclui as farinhas do macarrão propriamente dito, o macarrão instantâneo e o macarrão proveniente da sopa; a categoria massas inclui pizza, lasanha e massas de tortas; bolo inclui o bolo simples e a categoria cuscuz é constituída pelo cuscuz de milho seco e o cuscuz de milho com leite. Os outros alimentos, como pão de milho, broa de milho, mingau de fubá, farofa de milho e polenta (angu) não foram incluídos porque $90 \%$ ou mais das entrevistadas relataram o seu consumo como raro.

Tabela 1. Caracterização das gestantes atendidas em hospital universitário, em dois momentos. Brasília (DF), 2004, 2005.

\begin{tabular}{|c|c|c|c|c|c|c|c|c|c|c|}
\hline \multirow{2}{*}{ Variáveis } & \multicolumn{3}{|c|}{ Momento 1} & \multicolumn{3}{|c|}{ Momento 2} & \multicolumn{3}{|c|}{ Total } & \multirow{2}{*}{$p$-valor* } \\
\hline & $\mathrm{M}$ & DP & (IC95\%) & M & DP & (IC95\%) & $\mathrm{M}$ & $\mathrm{DP}$ & (IC95\%) & \\
\hline Idade (anos) & 26,4 & 5,8 & $(25,6-27,1)$ & 26,60 & 5,7 & $(25,8-27,3)$ & 26,5 & 5,7 & $(25,9-27,0)$ & 0,728 \\
\hline Idade gestacional (semanas) & 24,4 & 9,7 & $(23,2-25,7)$ & 24,20 & 9,4 & $(22,9-25,4)$ & 24,3 & 9,5 & $(23,4-25,2)$ & 0,766 \\
\hline Gestações (n) & 2,3 & 1,5 & $(2,1-2,5)$ & 2,20 & 1,4 & $(2,0-2,4)$ & 2,3 & 1,4 & $(2,1-2,4)$ & 0,323 \\
\hline Anos de estudo & 10,3 & 2,5 & $(9,9-10,6)$ & 10,70 & 2,6 & $(10,4-11,0)$ & 10,5 & 2,6 & $(10,2-10,7)$ & 0,072 \\
\hline Renda per capita $(\mathrm{R} \$)$ & 408,1 & 378,3 & $(358,2-458,1)$ & 468,12 & 350,1 & $(422,2-514,0)$ & 438,2 & 365,2 & $(404,5-472,2)$ & 0,082 \\
\hline Renda $\mathrm{SM}^{1}$ & 5,0 & 4,7 & $(4,4-5,6)$ & 5,00 & 4,1 & $(4,5-5,6)$ & 5,0 & 4,4 & $(4,6-5,4)$ & 0,956 \\
\hline Peso $(\mathrm{kg})$ & 64,2 & 12,9 & $(62,5-65,9)$ & 66,30 & 13,3 & $(64,6-68,1)$ & 65,3 & 13,1 & $(64,1-66,5)$ & 0,086 \\
\hline Altura $(\mathrm{cm})$ & 158,8 & 6,8 & $(157,9-159,7)$ & 159,10 & 6,0 & $(158,3-159,9)$ & 159,0 & 6,4 & $(158,4-159,5)$ & 0,602 \\
\hline IMC $\left(\mathrm{kg} / \mathrm{m}^{2}\right)$ & 25,4 & 4,6 & $(24,8-26,0)$ & 26,20 & 4,9 & $(25,6-26,8)$ & 25,8 & 4,8 & $(25,4-26,3)$ & 0,089 \\
\hline
\end{tabular}

M: média; DP: desvio-padrão.

* p-valor resultante do teste " $\mathrm{t}$ " de Student; ${ }^{2}$. Salário mínimo de acordo com o valor referente aos anos 2004 e 2005, respectivamente; Hipótese Nula $\left(H_{0}\right) \rightarrow$ As médias das variáveis idade, idade gestacional, número de gestações, anos de estudo, renda per capita, renda em salários mínimos, peso, altura e índice de massa corporal (IMC) são iguais entre os dois momentos; Hipótese Alternativa $\left(H_{1}\right) \rightarrow A_{s}$ médias das variáveis idade, idade gestacional, número de gestações, anos de estudo, renda per capita, renda em salários mínimos, peso, altura e IMC não são iguais entre os dois momentos. 
154 | I.A.L. VASCONCELOS et al.

Tabela 2. Consumo per capita total das farinhas e consumo equivalente às farinhas dos principais subprodutos, por gestantes atendidas em hospital universitário em dois momentos. Brasília (DF), 2004, 2005.

\begin{tabular}{|c|c|c|c|c|c|c|c|}
\hline \multirow{2}{*}{ Veículo/categoria } & \multicolumn{3}{|c|}{ Momento 1} & \multicolumn{3}{|c|}{ Momento 2} & \multirow{2}{*}{$p$-valor* } \\
\hline & M & DP & IC $95 \%$ & M & $\mathrm{DP}$ & IC $95 \%$ & \\
\hline Per capita farinha de trigo & 107,25 & 65,4 & $98,7-115,8$ & 101,51 & 60,9 & $93,6-109,5$ & 0,333 \\
\hline Pães de trigo & 57,30 & 45,5 & $51,4-63,3$ & 56,20 & 45,4 & $50,2-62,1$ & 0,783 \\
\hline Biscoitos & 15,95 & 23,8 & $12,85-19,1$ & 15,70 & 20,4 & $13,1-18,4$ & 0,909 \\
\hline Macarrão & 20,20 & 20,0 & $17,6-22,8$ & 18,20 & 18,3 & $15,8-20,6$ & 0,269 \\
\hline Massas & 6,30 & 13,4 & $4,6-8,1$ & 5,50 & 6,8 & $4,6-6,4$ & 0,405 \\
\hline Bolo & 5,60 & 9,8 & $4,3-6,9$ & 3,90 & 5,6 & $3,1-4,6$ & 0,021 \\
\hline Per capita farinha de milho & 14,50 & 23,9 & $11,3-17,6$ & 17,98 & 35,2 & $13,4-22,6$ & 0,212 \\
\hline Cuscuz & 12,60 & 22,6 & $9,6-15,5$ & 15,62 & 34,2 & $11,2-20,1$ & 0,264 \\
\hline Per capita de farinha total & 121,70 & 71,9 & $112,3-131,1$ & 119,50 & 76,1 & $109,6-129,4$ & 0,749 \\
\hline
\end{tabular}

M: média; DP: desvio-padrão.

* $p$-valor resultante do teste " $\mathrm{t}$ " de Student; Hipótese Nula $\left(\mathrm{H}_{0}\right) \rightarrow$ As médias das variáveis per capita farinha de trigo, pães de trigo, biscoitos, macarrão, massas, bolo, per capita farinha de milho, cuscuz e per capita de farinha total são iguais entre os dois momentos. Hipótese Alternativa $\left(\mathrm{H}_{1}\right) \rightarrow$ As médias das variáveis per capita farinha de trigo, pães de trigo, biscoitos, macarrão, massas, bolo, per capita farinha de milho, cuscuz, per capita de farinha total não são iguais entre os dois momentos.

Os alimentos de cada categoria que mais contribuíram para o consumo de farinhas foram o pão francês (medianas $\rightarrow 1^{\circ}$ momento: 30,0 e $2^{\circ}$ momento: 35,0 ), o biscoito salgado (medianas $\rightarrow 1^{\circ}$ momento: 6,99 e $2^{\circ}$ momento: 6,18$)$, o macarrão comum (medianas $\rightarrow 1^{\circ}$ momento: 6,74 e $2^{\circ}$ momento: 5,18 ) e a pizza (medianas $\rightarrow$ $1^{\circ}$ momento: 2,12 e $2^{\circ}$ momento: 2,36$)$. 0 consumo per capita total médio diário de farinhas foi estimado em $121,7 \mathrm{~g}$ no $1^{\circ}$ momento e $119,5 \mathrm{~g}$ no $2^{\circ}$ momento, com maior contribuição das farinhas de trigo em comparação às farinhas de milho. Não houve diferença significante em relação ao consumo de farinha de trigo, farinha de milho e o per capita total entre os dois momentos do estudo.

A média de porções diárias de cereais consumidas pelas gestantes foi estimada em 6,29 (Desvio-padrão- $\mathrm{DP}=3,04$; IC 95\%= 5,89-6,69), no $1^{\circ}$ momento, e 6,13 ( $D P=3,27$; IC 95\%=5,71-6,56), no $2^{\circ}$ momento. Desse total de porções, cerca de $65 \%$, em ambos os momentos, corresponderam a alimentos com farinha de trigo ou de milho na sua composição. $\mathrm{O}$ arroz, alimento mais consumido do grupo de cereais, apresentou uma média de porções de 1,42 (DP=0,85; IC 95\%=1,30 -1,53) e de 1,48 ( $D P=1,06$; IC 95\%=1,34-1,61), similar às porções consumidas de pão francês 1,30 $(D P=1,25$; IC $95 \%=1,14-1,46)$ e 1,32 (DP=1,28;
IC $95 \%=1,15-1,48)$, nos $1^{\circ}$ e $2^{\circ}$ momentos, respectivamente.

A Tabela 3 apresenta a distribuição das gestantes em relação às freqüências de consumo dos principais alimentos à base de farinhas nos dois momentos. As freqüências foram agrupadas em: consumo de, pelo menos, cinco vezes por semana (que inclui também o consumo diário); consumo entre uma a quatro vezes por semana; consumo entre uma a três vezes por mês; e consumo menor que uma vez por mês, definido como raramente ou nunca.

O pão francês foi o mais freqüentemente consumido, com ingestão por, pelo menos, cinco vezes na semana para mais de $50,0 \%$ das gestantes. Biscoitos, macarrão, bolo e cuscuz de milho foram consumidos pela maior parte das gestantes acima de quatro vezes por semana e as freqüências de consumo do sanduíche e da sopa com macarrão ficaram mais divididas entre uma a quatro vezes por semana (Sanduíche: 22,4\% e 24,6\%, Sopa com macarrão: $31,6 \%$ e $33,3 \%$, nos primeiro e segundo momentos, respectivamente) ou consumo mensal (Sanduíche: 30,3\% e $26,8 \%$, Sopa com macarrão: $28,9 \%$ e $30,7 \%$, no primeiro e no segundo momentos, respectivamente). O consumo da pizza se mostrou importante na freqüência mensal em ambos os momentos. 
Tabela 3. Distribuição de gestantes atendidas em hospital universitário quanto à freqüência qualitativa de consumo dos principais alimentos que contêm veículos de fortificação, em dois momentos. Brasília, (DF), 2004, 2005.

\begin{tabular}{|c|c|c|c|c|c|c|c|c|c|c|}
\hline \multirow{3}{*}{ Alimentos/Freqüência } & \multicolumn{2}{|c|}{ Momento 1} & \multicolumn{2}{|c|}{ Momento 2} & \multirow{3}{*}{$p$-valor } & \multicolumn{2}{|c|}{ Momento 1} & \multicolumn{2}{|c|}{ Momento 2} & \multirow{3}{*}{$p$-valor } \\
\hline & $n$ & $\%$ & $n$ & $\%$ & & $n$ & $\%$ & $n$ & $\%$ & \\
\hline & \multicolumn{4}{|c|}{ Pão Francês } & & \multicolumn{4}{|c|}{ Biscoito } & \\
\hline$\geq 5$ vezes/semana & 127 & 55,7 & 125 & 54,8 & $0,292^{2}$ & 49 & 21,5 & 45 & 19,7 & $0,849^{2}$ \\
\hline $1-4$ vezes/semana & 89 & 39,0 & 82 & 36,0 & & 119 & 52,2 & 128 & 56,2 & \\
\hline 1-3 vezes/mês & 3 & 1,3 & 9 & 4,0 & & 30 & 13,1 & 26 & 11,4 & \\
\hline$<1$ vez/ mês ${ }^{1}$ & 9 & 4,0 & 12 & 5,2 & & 30 & 13,1 & 29 & 12,7 & \\
\hline \multirow[t]{2}{*}{ Total } & 228 & 100,0 & 228 & 100,0 & & 228 & 100,0 & 228 & 100,0 & \\
\hline & \multicolumn{4}{|c|}{ Macarrão } & \multicolumn{6}{|c|}{ Bolo } \\
\hline$\geq 5$ vezes/semana & 11 & 4,8 & 14 & 6,1 & $0,346^{2}$ & 7 & 3,1 & 5 & 2,2 & $0,851^{2}$ \\
\hline $1-4$ vezes/semana & 149 & 65,4 & 136 & 59,7 & & 112 & 49,1 & 107 & 46,9 & \\
\hline 1-3 vezes/mês & 41 & 18,0 & 55 & 24,1 & & 67 & 29,4 & 74 & 32,5 & \\
\hline \multirow[t]{2}{*}{$<1$ vez/ mês ${ }^{1}$} & 27 & 11,8 & 23 & 10,1 & & 42 & 18,4 & 42 & 18,4 & \\
\hline & \multicolumn{4}{|c|}{ Cuscuz de milho } & \multicolumn{6}{|c|}{ Sopa com macarrão } \\
\hline$\geq 5$ vezes/semana & 6 & 2,6 & 9 & 4,0 & $0,781^{2}$ & 4 & 1,8 & 2 & 0,9 & $0,834^{3}$ \\
\hline $1-4$ vezes/semana & 102 & 44,8 & 94 & 41,2 & & 76 & 33,3 & 72 & 31,6 & \\
\hline 1-3 vezes/mês & 52 & 22,8 & 56 & 24,5 & & 66 & 28,9 & 70 & 30,7 & \\
\hline \multirow[t]{2}{*}{ <1 vez/ mês ${ }^{1}$} & 68 & 29,8 & 69 & 30,3 & & 82 & 36,0 & 84 & 36,8 & \\
\hline & \multicolumn{4}{|c|}{ Sanduíche } & \multicolumn{6}{|c|}{ Pizza } \\
\hline$\geq 5$ vezes/semana & 3 & 1,3 & 2 & 0,9 & $0,794^{3}$ & 1 & 0,4 & 0 & 0,0 & $0,434^{3}$ \\
\hline $1-4$ vezes/semana & 51 & 22,4 & 56 & 24,6 & & 20 & 8,8 & 21 & 9,2 & \\
\hline 1-3 vezes/mês & 69 & 30,3 & 61 & 26,7 & & 97 & 42,5 & 110 & 48,3 & \\
\hline$<1$ vez/ mês ${ }^{1}$ & 105 & 46,0 & 109 & 47,8 & & 110 & 48,3 & 97 & 42,5 & \\
\hline
\end{tabular}

${ }^{1}<1$ vez/ mês é corresponde à freqüência raramente ou nunca; ${ }^{2} p$-valor resultante do teste qui-quadrado, Graus de Liberdade $=3 ;{ }^{3} p$-valor resultante do teste exato do qui-quadrado, Graus de Liberdade $=3$.

Não houve diferença estatisticamente significante em relação à freqüência de consumo desses alimentos entre os dois momentos do estudo, o que demonstra o padrão alimentar similar entre as gestantes pesquisadas. Os outros alimentos, como pão de milho, broa de milho, mingau de fubá, farofa de milho, polenta (angu), lasanha e tortas não foram incluídos na tabela, porque $70,0 \%$ ou mais das entrevistadas relataram o consumo raro ou nunca consumiram.

A ingestão média diária dos principais nutrientes foi estimada também para os dois momentos e apresentada na Tabela 4. Não houve diferença significante entre os tipos de nutrientes e as quilocalorias consumidas entre os momentos, com exceção do consumo de ferro total $(p<0,0001)$ e do folato total $(p<0,0001)$. Para a obtenção de informações sobre esses dois nutrientes, além da comparação feita entre o consumo de ferro dietético nos dois momentos, comparou-se também o consumo de ferro e o de ácido fólico dietético do $1^{\circ}$ momento com o somatório do consumo dos nutrientes dietético e a quantidade proveniente da fortificação do $2^{\circ}$ momento - ferro adicional e ácido fólico adicional teórico. Para o cálculo do ácido fólico, considerouse o mínimo exigido pela legislação: $150 \mu \mathrm{g}$ por $100 \mathrm{~g}$ de farinha.

Para o $1^{\circ}$ momento, os alimentos hortaliças verde-escuras, biscoito, carne branca, carne vermelha, macarrão, feijão, pão e vísceras explicaram de forma significante $(p<0,0001)$ o ferro total antes da fortificação $\left(R^{2}=0,7846\right)$. Já no $2^{\circ}$ momento, considerando o ferro adicional teórico proveniente da fortificação, os alimentos biscoito, bolo, carne vermelha, cuscuz de milho, macarrão, feijão, pão de trigo e vísceras explicaram o ferro total $\left(R^{2}=0,8376\right)(p<0,0001)$ (Tabela 5). 
156 | I.A.L. VASCONCELOS et al.

Tabela 4. Ingestão média diária de energia e nutrientes em gestantes atendidas em hospital universitário, em dois momentos. Brasília (DF), 2004, 2005.

\begin{tabular}{|c|c|c|c|c|c|c|c|c|}
\hline \multirow{2}{*}{ Energia/Nutriente } & \multicolumn{3}{|c|}{ Momento 1} & \multicolumn{3}{|c|}{ Momento 2} & \multirow{2}{*}{$p$-Valor* } & \multirow{2}{*}{ Recomendação"I' } \\
\hline & M & DP & IC $95 \%$ & M & DP & IC 95\% & & \\
\hline Energia (Kcal) & 2722,00 & 1167,0 & $2570-2874,00$ & 2650,0 & 112,0 & $2504-2796$ & 0,503 & $2500 \mathrm{Kcal}$ \\
\hline Proteína (g) & 102,60 & 43,5 & $96,9-108,20$ & 103,6 & 44,5 & $97,8-109,4$ & 0,804 & $71 \mathrm{~g}$ \\
\hline Gordura (g) & 92,20 & 47,5 & $86,0-98,40$ & 91,3 & 46,6 & $85,2-97,4$ & 0,813 & - \\
\hline Carboidrato (g) & 374,10 & 165,4 & $352,2-395,70$ & 358,0 & 153,5 & $338,0-378,1$ & 0,282 & $175 \mathrm{~g}$ \\
\hline Vitamina A $(\mu \mathrm{g})$ & 3185,00 & 4736,6 & $2566,9-3803,10$ & 2919,5 & 2696,6 & $2567,6-3271,4$ & 0,462 & $800 \mu \mathrm{g}$ \\
\hline Vitamina $B_{1}(\mathrm{mg})$ & 2,30 & 1,4 & $2,1-2,50$ & 2,3 & 1,0 & $2,1-2,4$ & 0,918 & $1,4 \mathrm{mg}$ \\
\hline Vitamina $B_{2}(\mathrm{mg})$ & 2,70 & 2,4 & $2,4-3,00$ & 2,6 & 1,4 & $2,4-2,8$ & 0,535 & $1,4 \mathrm{mg}$ \\
\hline Niacina (mg) & 26,50 & 13,1 & $24,8-28,20$ & 27,0 & 13,2 & $25,3-28,7$ & 0,673 & $18 \mathrm{mg}$ \\
\hline Ácido Fólico dietético $(\mu \mathrm{g})$ & 301,98 & 194,6 & $276,6-327,40$ & 295,2 & 162,2 & $274,1-316,4$ & 0,688 & $355 \mu \mathrm{g}$ \\
\hline Folato total' $(\mu \mathrm{g})$ & 301,98 & 194,6 & $276,6-327,40$ & 474,5 & 234,9 & $443,8-505,1$ & 0,000 & $355 \mu \mathrm{g}$ \\
\hline Vitamina C (mg) & 299,90 & 309,5 & $259,4-340,30$ & 294,6 & 305,1 & $254,8-334,4$ & 0,854 & $55 \mathrm{mg}$ \\
\hline Cálcio (mg) & 991,99 & 476,1 & $929,9-1054,10$ & 988,8 & 501,7 & $923,3-1054,3$ & 0,944 & $1200 \mathrm{mg}$ \\
\hline Ferro dietético (mg) & 18,30 & 8,9 & $17,1-19,40$ & 18,4 & 8,3 & $17,3-19,5$ & 0,861 & $27 \mathrm{mg}$ \\
\hline Ferro total" (mg) & 18,30 & 8,9 & $17,1-19,40$ & 23,4 & 10,3 & $22,1-24,8$ & 0,000 & $27 \mathrm{mg}$ \\
\hline Zinco (mg) & 10,30 & 5,4 & $9,6-11,00$ & 11,2 & 6,3 & $10,3-12,0$ & 0,110 & $11 \mathrm{mg}$ \\
\hline
\end{tabular}

M: média; DP: desvio-padrão.

* valor-p resultante do teste "t"de Student; 'ácido fólico dietético + ácido fólico teórico da fortificação; " ferro dietético + ferro teórico da fortificação; "'Recomendação de energia de acordo com a RDA (1989)24; proteína e carboidrato de acordo com a IDR ${ }^{25}$ e micronutrientes segundo regulamentação da ANVISA ${ }^{26}$; Hipótese Nula $\left(H_{0}\right) \rightarrow$ As médias das variáveis relacionadas aos nutrientes/energia são iguais entre os dois momentos; Hipótese Alternativa $\left(H_{1}\right) \rightarrow$ As médias das variáveis relacionadas aos nutrientes/ energia não são iguais entre os dois momentos.

Tabela 5. Contribuição quantitativa de ferro proveniente de alimentos fontes e de alimentos sujeitos à fortificação em gestantes em dois momentos. Brasília (DF), 2004, 2005.

\begin{tabular}{|c|c|c|c|c|c|c|c|c|c|c|c|c|c|}
\hline & \multicolumn{6}{|c|}{ Momento 1 - Fe (mg) } & & \multicolumn{6}{|c|}{ Momento 2 - Fe (mg) } \\
\hline & $\mathrm{M}$ & DP & IC $95 \%$ & Valor $t$ & $p$ & $\mathrm{R}^{2}$ & & $\mathrm{M}$ & $\mathrm{DP}$ & IC $95 \%$ & Valor $t$ & $p$ & $\mathrm{R}^{2}$ \\
\hline Biscoito & 0,66 & 1,02 & $0,53-0,80$ & 4,10 & \multirow{8}{*}{$\begin{array}{l}\overline{8} \\
8 \\
\circ \\
\bar{v}\end{array}$} & \multirow{8}{*}{ 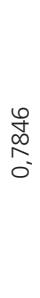 } & Biscoito & 1,31 & 1,70 & $1,09-1,53$ & 10,74 & \multirow{8}{*}{$\begin{array}{l}\bar{\delta} \\
8 \\
0 \\
\text { v }\end{array}$} & \multirow{8}{*}{$\begin{array}{l}0 \\
\infty \\
\infty \\
0\end{array}$} \\
\hline Hortaliça verde-escura & 0,20 & 0,31 & $0,16-0,24$ & 5,48 & & & Bolo & 0,41 & 0,59 & $0,33-0,48$ & 5,67 & & \\
\hline Carne branca & 0,78 & 0,71 & $0,68-0,87$ & 6,26 & & & Cuscuz de milho & 0,74 & 1,62 & $0,53-0,95$ & 12,01 & & \\
\hline Carne vermelha & 2,28 & 2,14 & $2,00-2,56$ & 10,00 & & & Carne vermelha & 2,61 & 2,86 & $2,24-2,99$ & 8,28 & & \\
\hline Macarrão & 0,37 & 0,39 & $0,32-0,42$ & 6,68 & & & Macarrão & 0,92 & 1,00 & $0,78-1,05$ & 5,10 & & \\
\hline Feijão & 1,59 & 1,49 & $1,40-1,79$ & 9,63 & & & Feijão & 1,52 & 1,50 & $1,33-1,72$ & 7,69 & & \\
\hline Pão & 1,07 & 0,90 & $0,95-1,19$ & 4,71 & & & Pão & 3,44 & 2,83 & $3,07-3,81$ & 13,56 & & \\
\hline Vísceras & 1,16 & 2,81 & $0,79-1,53$ & 11,44 & & & Vísceras & 0,87 & 1,41 & $0,69-1,06$ & 5,75 & & \\
\hline
\end{tabular}

M: média; DP: desvio-padrão.

\section{DISCUSSÃ O}

A medida regulatória que estabelece a obrigatoriedade da fortificação das farinhas de trigo e de milho entrou em vigor em junho de 2004. A partir de então, as farinhas passaram a ser obrigatoriamente fortificadas e a disponibilidade para o mercado consumidor se fez gradativamente, já que as farinhas previamente fabricadas ainda poderiam ser comercializadas até que acabasse o último lote. Por isso, não houve controle sobre quando as gestantes começaram a consumir os alimentos que contivessem as farinhas já fortificadas, o que faz disso uma limitação do estudo.

Além disso, a quantidade de ferro e o tipo de composto de ferro, adicionados pelo fabricante no $2^{\circ}$ momento da pesquisa, também não foram controlados. A legislação brasileira apenas determina a quantidade de ferro mínima a ser adicionada de $4,2 \mathrm{mg}$ de ferro por $100 \mathrm{~g}$ de farinhas. Dessa forma, o fabricante poderia adicionar mais ferro, 
e estar de acordo com a legislação, ou o teor de ferro poderia estar abaixo dos padrões estabelecidos pela resolução.

O Instituto Nacional de Metrologia, Normalização e Qualidade Industrial (INMETRO) ${ }^{30} \mathrm{fez}$ uma pesquisa pontual de análise do teor de ferro em seis marcas de fubás em junho de 2005 adquiridas em supermercados de Minas Gerais, do Rio de Janeiro, do Paraná. Foi constatado que duas marcas não estavam conforme o estabelecido pela legislação - considerando uma tolerância de $20 \%$ para mais ou para menos -, três marcas apresentaram teores cerca de $15 \%$ menores e uma marca continha $4,71 \mathrm{mg} / 100 \mathrm{~g}$ de farinha. Segundo o Instituto, somente a certificação e o acompanhamento regular assegurariam que o produto estivesse de acordo com os requisitos estabelecidos.

A legislação ainda permite a escolha do tipo de ferro pelo fabricante, o que também dificulta o controle da biodisponibilidade, já que não houve informação disponível sobre o tipo de composto utilizado pela indústria alimentícia.

Em uma dieta balanceada para as gestantes, recomenda-se de cinco a nove porções diárias de alimentos à base de cereais, segundo as recomendações do Guia Alimentar para a População Brasileira, do Ministério da Saúde ${ }^{31}$.

Estima-se que o peso da porção média de cereais seria de $64 \mathrm{~g}$, sendo que neste estudo cerca de $65 \%$ da quantidade de porções, em ambos os momentos, corresponderam a alimentos com farinha de trigo ou de milho na sua composição. Assim, seria recomendado o consumo por volta de $330 \mathrm{~g}$ de alimentos à base de farinhas de trigo ou milho por dia. Considerando os alimentos mais freqüentemente consumidos pelas entrevistadas, ou seja, pão de trigo, biscoito, bolo, macarrão e cuscuz, chegar-se-ia a uma média de $42 \%$ de teor de farinhas nesses alimentos.

Portanto, a recomendação de consumo per capita diário total de farinhas seria de, aproximadamente, $140 \mathrm{~g}$ de farinhas, número ligeiramente superior à média de consumo estimada para as gestantes do estudo. Se for considerado o valor mínimo de ferro proposto pela legislação brasileira, o aporte de ferro proveniente apenas da fortificação deve ser de, no mínimo, 5,9mg de ferro - aproximadamente $22 \%$ da Ingestão Dietética de Referência (IDR) ${ }^{26}$ para o período gestacional. Considerando as mesmas condições, as gestantes do estudo atingiram uma média de $5,1 \mathrm{mg}$ de ferro adicional, o que corresponde a $19 \%$ da IDR ${ }^{26}$.

A Pesquisa de Orçamento Familiar (POF) de 2002-2003, apesar de não ser um estudo que afere diretamente o consumo alimentar, mostrou que o gasto com a compra de cereais, leguminosas e oleaginosas na região Centro-Oeste foi um dos maiores do Brasil (13,4\%). Os gastos com farinhas, féculas e massas na mesma região foi de $4,2 \%$ e com panificados foi de 9,3\%. O pão francês foi um alimento habitualmente presente, porém, nas famílias com rendimentos mais baixos, a quantidade adquirida foi quase a metade da média nacional (12kg/ano), enquanto que nas com rendimentos mais elevados a quantidade foi $50,00 \%$ superior à média. No Distrito Federal (DF), a média anual per capita esteve acima de $12 \mathrm{~kg}^{32}$.

Além disso, segundo a POF 2002-2203, o macarrão não apresentou diferenças significantes de aquisição entre as regiões, sendo de $3,25 \mathrm{~kg} /$ ano no DF. O fubá de milho e a farinha de trigo apresentaram $1,14 \mathrm{~kg}$ e $1,35 \mathrm{~kg} / \mathrm{ano}$, respectivamente, sendo que este último alimento apresentou grande variação entre as regiões do País. A região Sul apresentou a média de consumo maior que 18kg. Comparando-se essa pesquisa com o Estudo Nacional de Despesa Familiar (ENDEF, 1974-1975) e as Pesquisas de Orçamento Familiares anteriores (1987-1988 e 1995-1996), considerando as diferenças metodológicas, observou-se que o consumo nacional de macarrão se manteve constante e o de pão francês apresentou uma diminuição constante de 22,0\% ao longo do tempo no País ${ }^{32}$.

Foi observado ainda que, considerando o ferro teórico adicional proveniente da fortificação, os alimentos à base de farinhas no $2^{\circ}$ momento da investigação tiveram maior contribuição no 
158 | I.A.L. VASCONCELOS et al.

consumo de ferro total em relação aos alimentos que são fontes naturais de ferro. Alimentos como hortaliças verde-escuras e carne branca, presentes como variáveis que explicam o consumo de ferro no $1^{\circ}$ momento, não apareceram no $2^{\circ}$ momento, em que o bolo simples e o cuscuz de milho se evidenciaram. O biscoito, o macarrão e o pão de trigo já explicavam o consumo de ferro total no $1^{\circ}$ momento, provavelmente em função da quantidade e da freqüência consumida e, no caso do biscoito, também por alguns tipos já serem enriquecidos com ferro voluntariamente pelo fabricante.

Mesmo com a fortificação considerada no $2^{\circ}$ momento do estudo, a média de ingestão de ferro não atingiu a recomendação para gestantes. Por isso, além da fortificação dos alimentos, outras práticas de prevenção e controle da anemia devem ser simultaneamente estimuladas. Isso inclui a educação nutricional, as orientações direcionadas ao consumo de alimentos com ferro mais biodisponível e o desestímulo ao consumo de inibidores da absorção do nutriente, além da suplementação, adotada como medida profilática na gestação. Foi constatado, nesta pesquisa, que apenas 34\% das gestantes estavam fazendo uso do suplemento de ferro durante a gestação, segundo o relato das próprias gestantes.

O nível de ingestão máxima tolerável (Tolerable Upper Intakes Levels) de ferro para gestantes, segundo o Institute of Medicine dos Estados Unidos, é de $45 \mathrm{mg} / \mathrm{dia}^{25}$. A comparação da quantidade de gestantes do $1^{\circ}$ e $2^{\circ}$ momentos que ultrapassaram esse limite, a partir da estimativa do consumo de ferro, não apresentou diferença significante (teste de Qui-quadrado, $p=0,431)$. Assim, a adição de ferro teórico estimada no $2^{\circ}$ momento não aumentaria significativamente a quantidade de gestantes que consumiram o nutriente acima do nível tolerável - 6 gestantes no $1^{\circ}$ momento $(51,1 \mathrm{mg}, \mathrm{DP}=4,8$; IC 95\%: 46,0-56,1) e 9 gestantes no $2^{\circ}$ momento (53,6mg, DP=7,5; IC 95\%: 47,8-59,4). Porém, deve-se considerar que a mensuração do ferro total por meio da análise do inquérito alimentar apenas proporciona a indicação de um risco. Na verdade, o risco do excesso de ferro pode ser mensurado com maior especificidade a partir da ferritina sérica².

Os resultados deste estudo mostram que os alimentos sujeitos à fortificação compulsória com ferro, mais especificamente aqueles que contêm como ingrediente a farinha de trigo, são veículos apropriados para a fortificação com ferro no que diz respeito ao largo consumo. Além disso, houve indicação de risco não significante de excesso de ingestão do nutriente pelas gestantes entrevistadas.

Recomenda-se a realização de outros estudos de consumo que considerem o aporte real adicional de ferro, a partir da mensuração direta do teor de ferro nesses alimentos, e que avaliem a biodiponibilidade dos compostos utilizados.

\section{A GRADECIMENTOS}

Ao professor Eduardo Freitas da Silva, do Departamento de Estatística da Universidade de Brasília, pelo auxílio nos cálculos e pela interpretação de dados estatísticos; ao apoio financeiro do Ministério da Saúde (FUNSAÚDE n² 2885/03); ao Observatório de Políticas de Segurança Alimentar e Nutrição (OPSAN), pelos recursos materiais e à Coordenadoria de Aperfeiçoamento de Pessoas de Nível Superior (CAPES), pela bolsa de fomento durante o mestrado.

\section{COLABORADORES}

M.H. CÔRTES E I.A.L. VASCONCELOS foram responsáveis pela revisão bibliográfica, pela coleta, pela análise e pela interpretação dos dados. D.C. COITINHO foi responsável pela concepção e pela orientação do estudo.

\section{REFERÊ NCIAS}

1. World Health Organization and Centers for Disease Control and Prevention. Assessing the iron status of populations: Report of a Joint World Health Organization, Centers for Disease Control and Prevention Technical Consultation on the Assessment of Iron Status at the Population Level. Geneva; 2004. 
2. World Health Organization. Iron deficiency anaemia: assessment, prevention and control. A guide for programme managers. Geneva; 2001. Document WHO/NHD/01.3.

3. Szarfarc SC. A anemia nutricional entre gestantes atendidas em centros de saúde do Estado de Säo Paulo (Brasil). Rev Saúde Pública. 1985; 19(5): 450-7.

4. Rodriguez OTS, Szarfarc SC, Benício MHA. Anemia e desnutrição maternas e sua relação com o peso ao nascer. Rev Saúde Pública. 1991; 25(3):193-7.

5. Nacul LC, Lira PI, Batista Filho M. Anemia em gestantes atendidas no pré-natal do IMIP. Rev Inst Materno Infantil de Pernambuco. 1991; 4(2):104-7.

6. Arruda IKG. Deficiência de ferro e folato e anemia em gestantes atendidas no IMIP: magnitude, alguns fatores de risco e repercussão nos seus conceptos [tese]. São Paulo: Programa de Pós-graduação em Nutrição da Universidade Federal de Pernambuco; 1997.

7. Food and Agriculture Organization. Food fortification: technology and quality control. Report of an FAO Technical Meeting, Rome, 20-23; 1995. FAO Food and Nutrition Paper. Rome: FAO; 1996.

8. Nestel P, Nalubola R, Sivakaneshan R, Wickramasinghe AR, Atukorala S, Wickramanayake $\mathrm{T}$. The use of iron-fortified wheat flour to reduce anemia among the estate population in Sri Lanka. Int J Vitam Nutr Res. 2004; 74(1):35-51.

9. Brasil. Resolução RDC $n^{\circ} 344$, de 13 de dezembro de 2002. Aprova o regulamento técnico para a fortificação das farinhas de trigo e das farinhas de milho com ferro e ácido fólico. [acesso 2004 fev]. Disponível em: <http://e-legis.bvs.br/leisref/public/ showAct. php?id=1679>.

10. Cintra IP, Heyde MED, Schmitz BAS, Franceschini SCC, Taddei JAAC, Sigulem DM. Métodos de inquéritos dietéticos. Cad Nutr. 1997; 13(2):11-23.

11. Brasil. Ministério da Saúde. Secretaria Executiva. Gestante de alto risco: sistema estaduais de referência hospitalar à gestante de alto risco. Brasília: Ministério da Saúde; 2001.

12. Côrtes MH. Impacto da fortificação das farinhas com ferro nos níveis de hemoglobina das gestantes atendidas pelo pré-natal do Hospital Universitário de Brasília [dissertação]. Brasília: Programa de Pós-Graduação em Nutrição Humana, Universidade de Brasília; 2006.

13. Vasconcelos FAG. Avaliação nutricional de coletividades. 3a. ed. Florianópolis: UFSC; 2000.

14. Atalah E, Castillo C, Castro R, Aldea A. Propuesta de un nuevo estándar de evaluación nutricional en embarazadas. Rev Méd Chile. 1997; 125(12): 1429-36.

15. Vítolo MR. Nutrição: da gestação à adolescência. Rio de Janeiro: Reichmann \& Affonso Editores; 2003.

16. Instituto Brasileiro de Geografia e Estatística. Censo Demográfico 2000. [acesso 2003 dez 10]. Disponível em: <http://www.ibge.gov.br/censo/ questionarios.shtm>.

17. Sávio KEO, Schmitz BAS. Perfil nutricional da clientela atendida em restaurantes vinculados ao Programa de Alimentação do Trabalhador do Distrito Federal: 2000-2001 [dissertação]. Brasília: Faculdade de Ciências da Saúde, Universidade de Brasília; 2002.

18. Zabotto CB, Veanna RPT, Gil MF. Registro fotográfico para inquéritos dietéticos: utensílios e porções. Goiânia: Unicamp; 1996.

19. Pinheiro ABV, Lacerda EMA, Benzecry EH, Gomes MCS, Costa VM. Tabela para avaliação de consumo alimentar em medidas caseiras. 4a. ed. São Paulo: Atheneu; 2000.

20. Moreira MA. Medidas caseiras no preparo dos alimentos. 2a. ed. Goiânia: AB Editora; 2002.

21. Erhardt J. Nutri survey for windows (software) 2005. University of Indonesia: SEAMEO-TROPMED; 2005. [cited 2005 Oct]. Available from: <http:// www.nutrisurvey.de/index.html>.

22. Philippi ST. Tabela de composição de alimentos: suporte para decisão nutricional. Brasília: ANVISA; 2001.

23. Instituto Brasileiro de Geografia e Estatística. Tabelas de composição de alimentos. 5a. ed. Rio de Janeiro: IBGE; 1999.

24. National Research Council. Recommended dietary allowances. 10th ed. Washington (DC): National Academy Press; 1989.

25. Institute of Medicine. Food and Nutrition Board. Dietary Reference Intakes. Washington (DC): National Academic Press; 1999-2001.

26. Brasil. Resolução RDC $n^{\circ} 269$, de 22 de setembro de 2005. O "Regulamento técnico sobre a ingestão diária recomendada (IDR) de proteína, vitaminas e minerais". [acesso 2006 jun]. Disponível em: $<$ http://e-legis.bvs.br/leisref/public/showAct. php?id=18828 $>$.

27. Statistical Package for the Social Science. SPSS 13.0 for windows. SPSS Inc; 2004

28. SAS. SAS 9.1 for windows. Cary (NC): SAS Inc; 2006.

29. Brasil. Ministério da Saúde. Conselho Nacional de Saúde. Comissão Nacional de Ética em Pesquisa. Diretrizes e Normas Regulamentadoras de 
Pesquisas em Seres Humanos. Resolução CNS No 196, de 10 de outubro de 1996. In: Normas para Pesquisa envolvendo seres humanos (Res. CNS n 196/96 e outras). 2a. ed. Brasília: Ministério da Saúde; 2003. p.29-51.

30. Instituto Nacional de Metrologia, Normalização e Qualidade Industrial (INMETRO). Relatório sobre análise em produtos de festa junina - amendoim, fubá de milho e leite de coco. Ministério do Desenvolvimento, Indústria e Comércio Exterior; 2005 [acesso 2005 jun]. Disponível em: <http:// www.inmetro.gov.br/consumidor/produtos/ festaJunina.asp $>$.
31. Brasil. Ministério da Saúde. Secretaria de Atenção à Saúde. Coordenação Geral da Política de Alimentação e Nutrição. Guia alimentar para a população brasileira: promovendo a alimentação saudável. Brasília: Ministério da Saúde; 2005.

32. Pesquisa de Orçamentos Familiares 2002-2003: primeiros resultados: Brasil e Grandes Regiões/ IBGE, Coordenação de índices de preços. Rio de Janeiro: IBGE; 2004.

Recebido em: 29/9/2006

Versão final reapresentada em: 1/11/2007 Aprovado em: 29/12/2007 